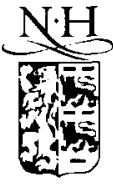

ELSEVIFR

\title{
Birefringence-induced losses in interferometers
}

\author{
W. Winkler, A. Rüdiger, R. Schilling, K.A. Strain ', K. Danzmann ${ }^{2}$ \\ Max-Planck-Institut für Quantenoptik, D-85748 Garching bei München, Germany
}

Received 17 May 1993; revised manuscript received 27 June 1994

\begin{abstract}
In interferometers one conceivable loss mechanism is depolarization of the light by inherent or thermally induced birefringence in optical substrates or coatings. The magnitude of this effect is determined quantitatively and compared with the losses due to thermal lensing.
\end{abstract}

\section{Introduction}

In some optical experiments it is essential to have low losses. One example is an interferometric gravitational wave detector, where recycling techniques are to be implemented [1,2]. Apart from losses due to absorption, some light may also be scattered into different geometric modes or coupled into other polarization states. The light may then be lost due to imperfect interference or due to some polarizing component, respectively. A discussion of relevant specifications of the surface figure (and implicitly the density fluctuations inside substrates traversed by the light) can be found in Refs. [3] and [4].

Thermal effects are likely to cause losses in high power experiments. Non-uniform thermal expansion and thermal lensing may deform the wavefront of the passing beams $[5,6]$, although part of the related losses can be recovered by signal recycling in an appropriately chosen optical lay-out $[7,8]$.

In the following sections we investigate the losses into different polarization modes through inherent or

\footnotetext{
Also at University of Glasgow.

2 Also at University of Hannover.
}

stress induced birefringence in both substrates and coatings. As an example, the results are eventually applied to an interferometric gravitational wave detector with power recycling, but without signal recycling.

\section{Inherent birefringence in substrates}

In the absence of any stress, most of the materials used as mirror and beamsplitter substrates have a spherical indicatrix, that is a constant index of refraction, independent of polarization and direction of propagation of the light.

Stresses deform the indicatrix, and the index of refraction becomes also polarization dependent. The stress may be introduced during the manufacturing process and frozen in - for instance because of the finite speed for cooling the material down. Many materials therefore show some inherent birefringence.

The inherent birefringence of fused silica - the most widely used substrate material - can be kept fairly small. Its magnitude may be defined by the phase difference $\delta$ introduced between light components polarized along orthogonal optical axes. Superposition of two such components can therefore produce ellipti- 
cally polarized light. Upper limits stated in the catalogue for Heraeus or Corning fused silica are $\delta=15$ $\mathrm{mrad}$ for green light and one $\mathrm{cm}$ of material traversed [9]. Inherent birefringence has been measured for the very homogeneous Corning 7940 grade 0A fused silica [10]: a $10 \mathrm{~cm}$ thick plate introduced a phase difference $\delta$ between the two orthogonal polarizations of up to $30 \mathrm{mrad}$; the values thus stay well below the upper limits quoted in the Heraeus catalogue. The inner parts of a larger sample are possibly even more homogeneous, especially with selection of the best batches. In this case $10 \mathrm{~cm}$ of material are likely to give a phase difference of less than $\delta=15 \mathrm{mrad}$.

In general the polarization of the input beam will not be parallel to one of the principal axes of the birefringent component, especially as the birefringence may vary locally. As a result, ellipticity, possibly position dependent, will be introduced. If so, any polarizing element in the system will remove part of the light. Assuming the worst case of input polarization oriented at an angle of $\phi=\pi / 4$ relative to the optical axes over the whole beam, the relative power loss per pass of the original polarization would be [11]:

$\frac{\Delta P}{P_{0}}=\sin ^{2} \frac{\delta}{2}$.

This relation gives an upper limit for the relative loss per pass of less than $10^{-4}$. Including such a component inside a cavity would still permit a power buildup of many thousands, if the inherent birefringence were the only round trip loss process.

\section{Thermally induced birefringence in substrates}

In addition to the inherent birefringence, stresses introduced in the experimental setup may also cause birefringence. Here we will deal with thermally induced stresses. They may result by inferring heat from outside into the optical components, or from heating by absorption of part of the illuminating light. We are mostly interested in the latter case.

\subsection{Stress distribution resulting from volume heating}

Koechner [12] presents a careful analysis of the birefringence in heavily pumped Nd:YAG laser rods. His formalism can be adapted for our purpose with only minor changes. He considers the case of a long laser rod, uniformly pumped throughout its volume, which is cooled along its cylindrical surface. Let us call this "volume heating". The radial heat flow gives rise to a quadratic temperature profile:

$T(r)=\frac{P_{\mathrm{a}}}{4 \pi r_{0}^{2} \kappa L}\left(r_{\mathrm{o}}^{2}-r^{2}\right)$,

with $r_{\mathrm{o}}$ the rod-radius, $P_{\mathrm{a}}$ the total heat dissipated in the rod, and $\kappa$ the heat conductivity of the rod material; the length of the rod is $L$. The temperature at the cylindrical surface was defined to be zero. The stress distribution inside the rod, as produced by this particular temperature profile, has the following radial, circumferential and longitudinal components:

$\sigma_{r}(r)=Q S\left(r^{2}-r_{0}^{2}\right)$,

$\sigma_{\phi}(r)=Q S\left(3 r^{2}-r_{\mathrm{o}}^{2}\right)$,

$\sigma_{z}(r)=2 Q S\left(2 r^{2}-r_{\mathrm{o}}^{2}\right)$.

The relevant quantities are $S=\alpha E /[16 \kappa(1-\nu)]$, $Q$ the density of absorbed power, $E$ Young's modulus, $\alpha$ the coefficient of thermal expansion, and $\nu$ Poisson's ratio.

\subsection{Magnitude of birefringence for volume heating}

Stresses generate strains in the rod, which in turn produce refractive index variations via the photoelastic effect. For our axisymmetric case we chose cylindrical coordinates with propagation in $z$-direction, the radial and tangential coordinates are $r$ and $\phi$, respectively.

Consider the plane perpendicular to the beam axis, with the $y$-axis oriented along the input polarization. Due to the axial symmetry of the thermal distortions, it is convenient to decompose the polarization at different points in the beam into a radial and a tangential component (see Fig. 1). The field at the $y$-axis has only a radial component, whereas the field at the $x$ axis has only a tangential component. Light propagating through these regions therefore remains linearly polarized. In other regions there are two components of polarization. These two components see a different, spatially varying change in their index of refraction due to the thermally induced strain. As a consequence there is a corresponding path difference introduced between the two components, leading to an ellipticity of the light-polarization. 


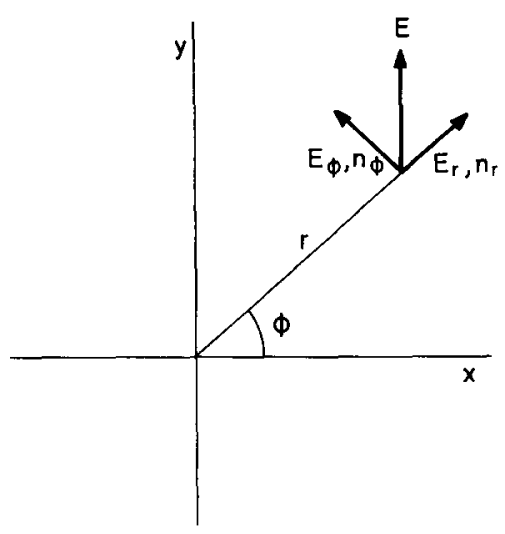

Fig. 1. Decomposition of the local electric field vector into its radial and tangential components.

For the case considered above, the relevant difference in index of refraction inside the birefringent component is

$\Delta n_{r}-\Delta n_{\phi}=n_{\mathrm{o}}^{3} \frac{\alpha Q}{\kappa} C_{\mathrm{B}} r^{2}$,

where the coefficient of birefringence, $C_{\mathrm{B}}$, is

$C_{\mathrm{B}}=\frac{1+\nu}{48(1-\nu)}\left(p_{11}-p_{12}+4 p_{44}\right)$.

$p_{i j}$ are the tensor components for the photoelastic effect; they can be found in the literature [13]. In isotropic media, $p_{44}$ is related to the two other relevant components by $2 p_{44}=p_{11}-p_{12}$ [11]. In this case we have

$C_{\mathrm{B}}=\frac{1+\nu}{16(1-\nu)}\left(p_{11}-p_{12}\right)$.

Fused silica is an amorphous material; it can be treated as intrinsically isotropic. The relevant numbers are $p_{11}=0.121$ and $p_{12}=0.270$. This gives $C_{\mathrm{B}}=-1.31 \times 10^{-2}$.

The phase difference between the two polarizations is given by

$\delta=\frac{2 \pi}{\lambda} L\left(\Delta n_{\phi}-\Delta n_{r}\right)$,

with $L$ the length of the rod. In an interferometer with some component sensitive to the polarization, for instance the beam splitter, light with polarization perpendicular to the preferred orientation will be partly removed. In case of a uniformly illuminated crosssection of the laser rod, Koechner [12] gets for the relative power per pass shifted out of the original polarization

$$
\frac{\Delta P}{P_{\mathrm{o}}}=\frac{1}{\pi r_{0}^{2}} \int_{\phi=0}^{2 \pi} \int_{r=0}^{r_{0}} \sin ^{2}(2 \phi) \sin ^{2} \frac{\delta}{2} r \mathrm{~d} r \mathrm{~d} \phi,
$$

where $\phi$ is the angle between one of the local optical axes and the $x$-axis. Integration yields

$\frac{\Delta P}{P_{\mathrm{o}}}=0.25\left[1-\operatorname{sinc}\left(2 C_{T} P_{\mathrm{a}}\right)\right]$,

where $C_{T}=2 n_{\mathrm{o}}^{3} \alpha C_{\mathrm{B}} / \lambda \kappa$. Sending a Gaussian beam through the rod with radius $w=r_{0} / 2$ instead of the uniform illumination, the depolarization losses are given by

$\frac{\Delta P}{P_{0}}=\frac{0.25}{1+16 / C_{T}^{2} P_{\mathrm{a}}^{2}}$.

\subsection{Temperature profile in a substrate heated by a Gaussian laser beam}

Eq. (12) was derived for a Gaussian laser beam sent through a substrate that was heated by uniform volume absorption of the pumping light, the corresponding temperature profile being quadratic, see Eq. (2). We are interested, however, in the thermal effects produced by non-uniform heating due to absorption of part of the transmitted laser beam itself. We will call this "beam heating". As we will see, Eq. (12) describes this situation quite well, provided the temperature drop across the beam radius is the same for both cases.

If a Gaussian laser beam is sent through a substrate with uniform absorption, a certain temperature profile inside the volume filled by the beam will form, and the entire optical component will be heated to some equilibrium state. The absorbed light power is radiated away by the whole substrate, if the heat conduction to the surrounding is small, as for components suspended inside a vacuum chamber. The radial temperature profile inside the absorbing component is given by [14]: 


$$
\begin{aligned}
& T(r)= \\
& \quad\left(\ln \frac{2 r_{\mathrm{a}}^{2}}{w^{2}}+C+\sum_{n=1}^{\infty} \frac{(-1)^{n}\left(2 r^{2} / w^{2}\right)^{n}}{n \cdot n !}\right) \frac{P_{\mathrm{a}}}{4 \pi \kappa L} \\
& =-\frac{P_{\mathrm{a}}}{4 \pi \kappa L}\left[E_{1}\left(\frac{2 r^{2}}{w^{2}}\right)+2 \ln \frac{r_{\mathrm{a}}}{r}\right],
\end{aligned}
$$

with the exponential integral $E_{1}(z)=\int_{z}^{\infty}\left(\mathrm{e}^{-t} / t\right) \mathrm{d} t$. Here a cylindrical surface parallel to and far from the beam axis is assumed. The temperature at this surface is taken as a reference and arbitrarily set to zero. The quantities used are $P_{\mathrm{a}}=$ total absorbed power, $\kappa=$ thermal conductivity, $L=$ thickness of the substrate, $r_{\mathrm{a}}$ = substrate radius, $w=$ beam radius, and $C=$ Euler's constant $(C \approx 0.577)$. The relation was derived by first solving the temperature distribution produced by heated hollow cylinders and then integrating over an infinite number of cylinders weighted with the Gaussian profile of the heating beam. End effects are neglected. The results agree with those obtained by Hello and Vinet through a different approach [5].

\subsection{Birefringence caused by beam heating}

To describe the power loss induced by birefringence, one has to start out with Eq. (10), and include the Gaussian intensity distribution. A rigorous calculation has to determine $\delta$ eventually as a function of the stress distribution inside the substrate. The just mentioned paper by Hello and Vinet contains exact, but fairly complicated, relations for stresses and strains in a beam-heated substrate. In order to get an estimate of the magnitude of the thermally induced birefringence, we choose a much simpler approach, sufficient for our purpose.

As the temperature gradients are responsible for internal stresses, leading to strains and eventually to birefringence, we choose for $\delta$ the functional dependence of the temperature distribution itself - first the quadratic (Koechner's) case and second the beam heating case, Eq. (13). Despite of the totally different functions included, the results differed only by $16 \%$, if the temperature drop $\Delta T$ from the beam axis over a distance of the beam radius $w$ was set equal. In Koechner's case, $\Delta T$ is:

$$
\Delta T=\frac{w^{2}}{r_{0}^{2}} \frac{P_{\mathrm{a}}}{4 \pi \kappa L},
$$

where, in his particular arrangement of a heated laser rod, $r_{0}=2 w$. For the case of heating by the beam itself we get

$$
\Delta T=1.3 \times \frac{P_{\mathrm{a}}}{4 \pi \kappa L} .
$$

The temperature drop is set equal for both cases, if the absorbed power $P_{\mathrm{a}}$ for Koechner's constant volume absorption is replaced by $1.3 \times\left(r_{\mathrm{o}} / w\right)^{2} \times P_{\mathrm{a}} \approx$ $5.2 \times P_{\mathrm{a}}$. This means that power absorbed out of the beam has a bigger effect on the beam itself than the same power uniformly absorbed in the whole volume, since it causes stronger temperature gradients across the beam volume.

As the deformation of the substrate surface [5] (showing to a great extent the internal stress distribution) obeys a function running between the two temperature functions which we inserted in Eq. (10), our estimate can be considered to describe the situation with an accuracy of the order of $10 \%$. For all practical purposes our simple correction to Koechner's equations is therefore sufficient to describe the case of a substrate heated by the passing laser beam. This close result is due to the fact that the temperature drop across the beam diameter determines the relevant strain distribution, and thus also the thermally induced birefringence. Contributions from outside the beam diameter are of minor importance. Variations in the uniformity of the absorption due to material inhomogeneities may limit the usefulness of more accurate calculations anyhow.

For high finesse cavities only small losses are tolerable and Eq. (12) may be expanded to give

$$
\frac{\Delta P_{\text {depol }}}{P_{\mathrm{o}}} \approx\left(\frac{1.3 n_{\mathrm{o}}^{3} \alpha C_{\mathrm{B}}}{\lambda \kappa} P_{\mathrm{a}}\right)^{2}
$$

It may be of some practical interest to compare this loss with the loss due to thermal lensing. The relative power which the thermal lens, produced by the same temperature profile, removes from the original fundamental geometrical mode of the light is given by Eq. (11) in Ref. [6]:

$$
\frac{\Delta P_{\text {th.l. }}}{P_{\mathrm{o}}} \approx\left(\frac{1.3 \beta}{4 \lambda \kappa} P_{\mathrm{a}}\right)^{2} \text {. }
$$

The ratio of Eqs. (16) and (17) gives 
Table 1

Limiting power $P_{\text {lim }}$ for losses due to birefringence or thermal lensing for some materials

\begin{tabular}{lll}
\hline Material & $P_{\text {lim,depol }}$ & $P_{\text {lim,th.l. }}$ \\
\hline fused silica & $30 \mathrm{~W}$ & $0.16 \mathrm{~W}$ \\
YAG & $15 \mathrm{~W}$ & $3 \mathrm{~W}$ \\
glass & $2 \mathrm{~W}$ & $1.5 \mathrm{~W}$ \\
\hline
\end{tabular}

$$
\frac{\Delta P_{\text {th.l. }}}{\Delta P_{\text {depol }}} \approx\left(\frac{\beta}{4 n_{\mathrm{o}}^{3} \alpha C_{\mathrm{B}}}\right)^{2} .
$$

For fused silica the thermal lens is by far dominating: the ratio is slightly bigger than $10^{4}$. For YAG crystals this ratio is approximately 15 , and for glass it is close to 1 .

Eqs. (16) and (17), describing the relative power loss $\triangle P / P$ due to thermal lensing and stress induced birefringence, can be parametrised in the form:

$\frac{\Delta P}{P_{\mathrm{o}}}=\left(\frac{P_{\mathrm{a}}}{P_{\mathrm{lim}}}\right)^{2}$,

with the limiting parameter $P_{\text {lim }}$ given in Table 1 .

Clearly, the absorbed power has to be much smaller than $P_{\text {lim }}$. An example: an absorption of $1.6 \mathrm{~mW}$ of light power produces a relative power loss of $10^{-4}$ by thermal lensing in fused silica; to get the same loss by birefringence, the absorbed power would have to be $300 \mathrm{~mW}$.

\section{Inherent birefringence in coatings}

The magnitude of birefringence in optical coatings can be defined by the phase difference $\delta$ introduced between two different polarizations after one reflection at the coating. The effect of birefringence in the coating is most critical for cavity mirrors. Let us therefore consider this case. There is no general criterium for the tolerable magnitude of birefringence. Often one wants the relative power loss inside the cavities to be dominated by the power transmission $t^{2}$ of the coupling mirrors. Power losses may result from shifting a relative amplitude of light $\Delta a / a$ at each reflection into the "wrong" polarization, and after eventually leaving the cavity, this light may be removed by some polarizing component. $\Delta a / a$ has therefore to be smaller than $t$. (For $\Delta a / a>t$ only one polarization can be made resonant inside the cavity; the other polarization can not enter the cavity. It is therefore sufficient to consider the case $\Delta a / a<t$ ). The ratio $\Delta a / a$ will depend on the orientation of the local principal axes, but an upper limit is given by $\Delta a / a=\delta / 2$ (see Eqs. (1) and (10)). For $\delta<t$ both polarizations are resonant simultaneously in the cavities. If the depolarization through birefringence were the only loss, and the two mirrors of the cavity gave the same contribution, then the effective reflectivity of the cavity would be

$\rho_{c} \approx 1-(\delta / t)^{2}$.

With no losses the reflectivity of the cavity would be equal to 1 . In the past the coatings produced by electron beam evaporation showed a relatively strong birefringence of up to one mrad [15]. This value has been reduced with the technique of ion-beam sputtering and improved geometry in the vacuum chamber for better homogeneity of the coating. The lowest values for $\delta$ obtainable today are between 2 to $10 \mu \mathrm{rad}$ per reflection, but so far only for mirrors and beams with a size of a few $\mathrm{cm}$ and $\mathrm{mm}$, respectively. [16] For larger components the values are not yet known.

\section{Thermally induced birefringence in coatings}

So far little is known about thermally induced birefringence in the coatings. Its magnitude may be estimated in the following way: The effect is related to the thermally induced strains inside of the material involved. The strains occur as a consequence of local heating either by absorption of some light in the coating or in the substrate traversed by the light. In both cases the dominating deformation results from an expansion of the hemisphere around the reflection spot, extending into the substrate by a dimension comparable to the beam size $[6,12]$. Absorption at regions inside the substrate further apart from the surface do not contribute noticeably to the local strain there. The coating follows the deformation of the substrate and consequently shows some birefringence.

Let us now consider the same case as in the last section, namely birefringence in the mirror coatings of Fabry-Pérot cavities, but the birefringence produced now by absorption of part of the illuminating light. This seems to be a most critical region, as the light power at the mirror coatings may be very high. As- 
sume the power absorbed in the coating to be $P_{\mathrm{a}}$. Birefringence will occur because of mechanical strains in the coatings. These strains result from a thermally induced expansion of the surface-near part of the substrate behind the reflection spot. The contribution of the temperature drop $\Delta T$ across the thickness $d$ of the coating does not contribute noticeably: the corresponding expansion $\Delta w$ of the coating along the surface over a distance of beam-radius $w$ can be estimated by starting from the equation for heat conduction through the coating:

$P_{\mathrm{a}}=\kappa_{\mathrm{coat}} A \nabla T \approx \kappa_{\mathrm{coat}} \pi w^{2} \frac{\Delta T}{d}$.

From this we get for the temperature drop across the thickness of the coating

$\Delta T \approx \frac{d}{\kappa \pi w^{2}} P_{\mathrm{a}}$.

The expansion of the radius $w$ of the reflection spot by $\Delta w$ is therefore

$\Delta w \approx\left(\frac{\alpha}{2 \pi \kappa}\right)_{\text {coat }} \frac{d}{w} P_{\mathrm{a}}$,

whereas the temperature gradient inside the substrate leads to an expansion of the hemisphere behind the reflection spot by [6]

$\Delta w \approx\left(\frac{\alpha}{4 \pi \kappa}\right)_{\text {substr }} P_{\mathrm{a}}$.

For fused silica we have $\alpha / \kappa=0.3 \times 10^{-6}$, and for $\mathrm{TiO}_{2}: \alpha / \kappa=1 \times 10^{-6}$, both values given for bulk material. In the past, the properties of coatings were not well defined. Sometimes the layers were not at all homogeneous, but had rather a columnar structure. The thermal conductivity could be orders of magnitude different from that of bulk material [18], and could also be anisotropic [19]. The high quality layers produced today by ion-beam sputtering are very homogeneous and have properties very close to those of the bulk material. Therefore the expansion of the substrate as described in Eq. (24) dominates the stress in the coating, whereas the contribution of Eq. (23) is negligible because of the small factor $d / w$.

In textbooks one often finds for an estimate of the magnitude of birefringence the simple equation [20]

$\Delta n=K \sigma$, where $\Delta n$ is the difference in index of refraction introduced in a material under a stress $\sigma$, and $K$ a constant specific for each material. Eq. (25) does not take into account the tensor character of the properties of a deformed material, but is sufficient for most purposes. In our case we may start an estimate from the temperature drop across the hemisphere around the reflection spot and the resulting thermal expansion, and then get for the relevant stress at the beam center [6]

$\sigma \approx \frac{\alpha}{4 \pi \kappa} \frac{E}{w} P_{\mathrm{a}}$,

with $E$ the elastic modulus of the substrate. From Eq. (1) one finds the maximum relative amplitude shifted into the wrong polarization to be $\Delta a / a \approx \delta / 2$, with

$\delta=2 \pi \frac{\Delta n d}{\lambda}$,

where $d$ is some effective thickness of the coating as seen by the light reflected at the surface; it may be estimated to be on the order of a few wavelengths (only the first few layers contribute noticeably, but they are traversed twice). In the worst case the whole crosssection of the beam contributes fully to the depolarization. The relative amplitude of light shifted into the wrong polarization at each reflection would then be given by

$\frac{\Delta a}{a}=\frac{\alpha K}{4 \kappa \lambda} \frac{d}{w} E P_{\mathrm{a}}$.

This relation shows that depolarization due to absorption-induced birefringence in the coating decreases with longer armlength: the effective thickness $d$ of the coating as seen by the reflected light is independent of the size of the components, whereas the beam radius $w$ grows with the square root of the mirror separation. Under the assumption of the previous sections, $\Delta a / a<t$, light in the unwanted polarization can build up inside the cavity; part of it will leak out according to the transmitance of the coupling mirror and may be lost at some polarizing element.

\section{Limitations for interferometric gravitational-wave detectors set by birefringence}

As already mentioned, one example for a high power laser interferometer is an interferometric gravitational wave antenna. Even though in currently 
existing prototypes birefringence has not been a problem, for the envisaged high light power and enhanced sensitivity the situation might change.

The optical arrangement of an interferometric gravitational wave detector will be adapted to the signals one will be looking for. One possibility is a Michelson interferometer with a Fabry-Pérot cavity in each arm to increase the optical pathlength. In addition there may be a mirror between the illuminating laser and the interferometer to recycle the light leaving the interferometer in the direction back to the laser [17]. This additional mirror is placed to form the so called recycling cavity together with the whole interferometer as a second mirror. The addition of signal recycling (another mirror at the output of the interferometer) complicates the situation; but its tendency is to suppress light in other than the fundamental mode [8]. It is sufficient for our purpose to consider an interferometer with power recycling only.

The tolerable magnitude of birefringence may be estimated by the limit it poses on the power enhancement $G$. The constant $G$ is defined as the ratio between the power built up inside the power recycling cavity, for example at the beam splitter, to the power delivered by the illuminating laser. In order to get a high $G$, the losses inside the power recycling cavity have to be kept small. Based on the relations obtained above, we may estimate the contribution of birefringence to these losses.

Inherent birefringence, either in substrates or in coatings, acts like a fixed loss. Substrates relevant here are the beam-splitter, the compensation plate and the coupling mirrors of the arm cavities.

Fused silica is the most widely used substrate material. As of today's state of the art, it would allow a recycling gain of up to about $10^{4}-$ a value sufficient even for advanced detectors (see Section 2, especially Eq. (1)).

The same statement is true for inherent birefringence in coatings, provided the coatings of the fairly large mirrors can be manufactured with the same specifications as presently possible for small ones. Most critical are the coatings in the arm cavities. For optimum coupling of the laser light into the recycling cavity the reflectivity of the power recycling mirror has to be equal to the reflectivity of the arm cavity (see Eq. (20) and assuming no extra losses). In this case, the power recycling factor $G$ is given by
$G=\frac{1}{2}(t / \delta)^{2}$

Birefringence of the coating is not dominating the loss budget for a desired power recycling factor $G$, if

$\delta<t / \sqrt{2 G}$.

The transmission $t$ of the coupling mirrors of the arm cavities is not expected to be smaller than, say, $10^{-2}$; a power recycling gain of $10^{4}$ may be the best one can hope for. Even for these extreme cases, inherent birefringence should not limit the performance of interferometric gravitational wave detectors, if the phase difference introduced at each reflection between the two states of polarizations can be kept at a level of the above mentioned $10 \mu \mathrm{rad}$.

Thermally induced birefringence in substrates has been described in Section 3. Eq. (16) gives the corresponding depolarization losses. In order not to be limiting for the recycling gain $G$, these losses have to be smaller than $1 / G$. The absorption in high quality fused silica is now only a few ppm per cm; recently Heraeus has produced a sample of fused silica with particularly low $\mathrm{OH}$-content, giving an absorption at a level of less than $1 \mathrm{ppm} / \mathrm{cm}$ [21] for light of $1 \mu \mathrm{m}$ wavelength.

Eq. (19) shows that a recycling gain of $G=10^{4}$ could be achieved with an absorbed light power of $300 \mathrm{~mW}$, if thermally induced depolarization were the dominating loss mechanism. But as mentioned above: thermal lensing is here the dominating effect; $300 \mathrm{~mW}$ of absorbed light power would already have rendered the recycling cavity unstable. Circulating light powers of $100 \mathrm{~kW}$ (or even more) can therefore not be realized with present technology; but with further reduced absorption and with suppression of higher modes by signal recycling (or signal extraction [2]) such values do not seem to be impossible.

Let us finally consider the effect of thermally induced birefringence in the coating. An estimate has been given by relation (28). For these losses not to limit the power build up in the recycling cavities, the power absorbed at each of the cavity mirrors has to be kept below a certain limit:

$P_{\mathrm{a}}<\left(\frac{\kappa}{\alpha E}\right)_{\text {substr }} 2 \lambda w\left(\frac{1}{K d}\right)_{\text {coat }} \frac{t}{\sqrt{2 G}}$.

Let us take the example of fused silica, coated with alternate layers of $\mathrm{TiO}_{2}$ and $\mathrm{SiO}_{2}$. For fused silica we 
have $K=3.7 \times 10^{-8} \mathrm{~cm}^{2} / \mathrm{N}$, and for $\mathrm{TiO}_{2}: K \approx$ $20 \times 10^{-8} \mathrm{~cm}^{2} / \mathrm{N}$. Let us again assume extreme conditions: For a power enhancement factor $G$ of $10^{4}$, a power transmission $t^{2}$ of $10^{-4}$, a beam radius of $2 \mathrm{~cm}$ and an effective thickness $d$ of the layers of $2 \mu \mathrm{m}$, the power absorbed in the coating of one cavity mirror needs to stay below about $1 \mathrm{~W}$. This is not too hard to fulfil, given currently available coatings which show an absorption on the order of $1 \mathrm{ppm}$. But as above, thermal lensing gives stronger restrictions; it appears now in the substrate because of absorption in the coating.

\section{Conclusion}

Quantitative relations have been given for the loss of light power from a particular state of polarisation by inherent or thermally induced birefringence, either in substrates or in coatings. Especially the thermal effect due to absorption of part of the illuminating light has been considered in detail. Loss by thermally induced birefringence may be fairly important; but during the last few years huge progress has been made in manufacturing extremely low absorbing substrates and coatings. The coatings made so far mostly had a diameter of less than a few $\mathrm{cm}$; little is known for coatings with larger diameters. Applying our results to high finesse cavities, and assuming the lowest values for absorption and inherent birefringence reported so far, we find that birefringence is not going to be the dominant loss mechanism, even in the case of intracavity components and very high light power. Thermal lensing is more critical, especially in the case of fused silica. It has been treated to some extent in Refs. $[5,6]$ and [7], and will be investigated in more detail in a following paper [8].

\section{Acknowledgements}

We want to thank Jennifer Logan from the Glasgow group and David Shoemaker from MIT for helpful discussions and for making available to us their results of measurements on fused silica.

\section{References}

[1] B.J. Meers, Phys. Rev. D 38 (1988) p. 2317.

[2] J. Mizuno, K.A. Strain, P.G. Nelson, J.M. Chen, R. Schilling, A. Rüdiger, W. Winkler and K. Danzmann, Phys. Lett. A 175 (1993) 273.

[3] W. Winkler, J. Chen, K. Danzmann, P.G. Nelson, T.M. Niebauer, A. Rüdiger, R. Schilling, K.A. Strain, L. Schnupp, H. Walther, J. Hough, A.M. Campbell, C.A. Cantley, J.E. Logan, B.J. Meers, E. Morrison, G.P. Newton, D.I. Robertson, N.A. Robertson, S. Rowan, K.D. Skeldon, P.J. Veitch, H. Ward, H. Welling, P. Aufmuth, I. Kröpke, D. Ristau, J.E. Hall, J.R.J. Bennett, I.F. Corbett, B.W.H. Edwards, R.J. Elsey, R.J.S. Greenhalgh, B.F. Schutz, D. Nichoison, J.R. Shuttleworth, J. Ehlers, P. Kafka, G. Schäfer, H. Braun and V. Kose, The Optics of an Interferometric Gravitational-Wave Antenna, in: Lecture Notes in Physics, Vol. 410 (Springer, Berlin, 1992) p. 210.

[4] W. Winkler, K. Danzmann, A. Rüdiger and R. Schilling, Optical Problems in Interferometric Gravitational Wave Antennas, in: Proceedings of the 6th Marcel Grossmann Meeting, Part A (Kyoto, 1991) p. 176.

[5] P. Hello and J.Y. Vinet, J. Phys. (France) 51 (1990) 2243.

[6] W. Winkler, K. Danzmann, A. Rüdiger and R. Schilling, Phys. Rev. A 44 (1991) 7022.

17] B.J. Meers and K.A. Strain, Phys. Rev. D 43 (1991) 3117.

[8] K.A. Strain, K. Danzmann, A. Ruidiger, R. Schilling and W. Winkler, Phys. Lett. A 194 (1994) 124.

[9] Quarzglas für die Optik, Daten und Eigenschaften, Heraeus, POL-O/101.

[10] J.E. Logan, Ph.D. Thesis, University of Glasgow (1993).

$111]$ M. Born, E. Wolf, Principles of Optics (Pergamon Press, Oxford, 1989).

[12] W. Koechner, Springer Series in Optical Sciences, SolidState Laser Engineering, Vol. 1 (Springer, Berlin, 1988) p. 350 .

[13] F.T. Arecchi and E.O. Schulz-Dubois, eds., Laser Handbook, Vol. 1 (North Holland, Amsterdam, 1972) p. 999.

|14| F. Meyer, Max-Planck-Institut für Astrophysik, private communication.

[15] M.A. Bouchiat and L. Pottier, Appl. Phys. B 29 (1982) 43.

[16] C. Wieman, JILA, private communication.

[17] R.W.P. Drever, in: Gravitational Radiation, eds. N. Deruelle and T. Piran (North Holland, Amsterdam, 1983) p. 321.

| $18 \mid$ J.C. Lambropoulos, J. Appl. Phys. 66 (1989) 4230.

[19] D. Ristau, Laser-Zentrum Hannover, private communication.

[20] Mütze, Foitzik, Krug and Schreiber, ABC der Optik (Werner Dausien, Hanau/Main, 1972) p. 812.

[21] N.C. Man, LAL Orsay, private communication. 